\title{
Multi-objective Geometric Programming with Varying Parameters: Application in Waste Water Treatment System
}

\section{Mrinal Jana and Geetanjali Panda}

\author{
Indian Institute of Technology Kharagpur \\ Kharagpur-721302, India \\ E-mail(corresp.): geetanjali@maths.iitkgp.ernet.in \\ E-mail: mrinal.jana88@gmail.com
}

Received April 23, 2015; revised August 18, 2015; published online September 15, 2015

\begin{abstract}
In this paper a general multi-objective geometric programming problem with interval parameters is proposed, and a methodology is developed to derive its solution. The model is transformed into a general geometric programming problem, and relation between the original problem and the transformed problem is established. Application of this discussion is illustrated in a waste water treatment model.
\end{abstract}

Keywords: mathematical model, nonlinear problem, order relation, multivalued mapping.

AMS Subject Classification: 90C29; 90C30; 90C90; 97M10; 97M40.

\section{Introduction}

Since last two decades, linear/non-linear interval optimization problems have been studied by many researchers (see $[1,2,7,8,9,9,10,11,12,13,18,28]$ ). Some of these models consider interval parameters in the objective function only. Most of the above papers focus on the derivation of optimal bounds of interval optimization models. The methodologies due to $[1,11]$ focus on the existence of solution of nonlinear interval optimization problems with several assumptions. In the literature of the theory of interval optimization, readers may observe the existence of very few number of research papers on interval geometric programming. The existing literature is limited to some particular type interval geometric programming models only. A single objective interval geometric (posynomial) programming model takes the following form.

$(I G P) \quad \min _{x} \sum_{t=1}^{s_{0}} \hat{c}_{0 t} \prod_{j=1}^{n} x_{j}^{\hat{a}_{0 t j}}$ subject to $\sum_{t=1}^{s_{i}} \hat{c}_{i t} \prod_{j=1}^{n} x_{j}^{\gamma_{0 t j}} \leq \hat{b}_{i}, i=1,2, \ldots, m$, $x_{j}>0, j=1,2, \ldots, n$, where $\hat{c}_{0 t}, \hat{a}_{0 t j}, \hat{c}_{i t}, \hat{b}_{i}, \forall i, j, t$ are intervals. 
Single objective geometric programming models with interval parameters $(I G P)$ are studied by $[3,8,15,16,17,20]$. Most of these methods formulate a pair of two-level mathematical programming problems as

$$
\begin{aligned}
\left(P_{1}\right) \quad \max _{\hat{S}} \min _{x} \quad \sum_{t=1}^{s_{0}} \hat{c}_{0 t} \prod_{j=1}^{n} x_{j}^{\hat{a}_{0 t j}} \\
\text { subject to } \quad \sum_{t=1}^{s_{i}} \hat{c}_{i t}\left(\hat{b}_{i}\right)^{-1} \prod_{j=1}^{n} x_{j}^{\gamma_{0 t j}} \leq 1, x_{j}>0, \\
\\
i=1,2, \ldots, m, j=1,2, \ldots, n
\end{aligned}
$$

and

$$
\begin{aligned}
\left(P_{2}\right) \quad \min _{\hat{S}} \min _{x} \quad & \sum_{t=1}^{s_{0}} \hat{c}_{0 t} \prod_{j=1}^{n} x_{j}^{\hat{a}_{0 t j}} \\
\text { subject to } \quad & \sum_{t=1}^{s_{i}} \hat{c}_{i t}\left(\hat{b}_{i}\right)^{-1} \prod_{j=1}^{n} x_{j}^{\gamma_{0 t j}} \leq 1, x_{j}>0 \\
& i=1,2, \ldots, m, j=1,2, \ldots, n
\end{aligned}
$$

where $\hat{S}$ is the set of all intervals present in the above models. Consequently optimal values of $P_{1}$ and $P_{2}$ provide lower and upper bound of the optimal value of $I G P$. But these methods do not provide the optimal solution of IGP.

There are several real life interval geometric programming models with more than one objective functions, known as multi-objective geometric programming model. One of these model related to waste water treatment system is explained in the last section of this paper. Multi-objective geometric programming with interval parameters has not been addressed yet. This motivated the authors to focus on the theory of general interval multi-objective geometric programming problem. It is obvious that the methodology for general multi-objective geometric programming problem may not work for interval multi-objective geometric programming problem due to the presence of interval uncertainty. Moreover, the methodologies described in $[3,8,15,16,17,20]$, for interval geometric programming, provide lower and upper bounds of the objective function at two different points, but do not focus on optimal solution. In this paper the authors have tried to meet these gaps. Initially, existence of solution of this model is studied. A methodology is derived to find its efficient solution, which can provide a compromising lower and upper bound for all objective functions. As a result, one can find a solution as well as optimal bounds. Throughout this paper $(I M G P)$ denotes an interval multi-objective geometric programming problem. A general $(I M G P)$ model is proposed in Section 3.

The paper is divided in four major sections. Section 2 explains some notations and prerequisites on interval analysis. Section 3 describes solution methodology for a general multi-objective geometric programming model. Section 4 describes the proposed waste water treatment model as an (IMGP) problem. This model is formulated through some hypothetical data. Methodology of Section 3 is used to solve this model. 


\section{Mathematical notations, concepts and definitions}

Following notations are used throughout the paper.

- $I(\mathbb{R})$ : The set of closed intervals on $\mathbb{R}$. $\hat{a} \in I(\mathbb{R})$ is the set $\hat{a}=\left[a^{L}, a^{R}\right]$, $\hat{a}$ is said to be a degenerate interval if $a^{L}=a^{R}$ and is denoted by $\widehat{A}$.

- $I(\mathbb{R})_{+}$: The set of positive closed intervals on $\mathbb{R}$, i.e., $\hat{a} \in I(\mathbb{R})_{+}$if $a^{L}>0$.

- $I(\mathbb{R})^{n}:$ The set of interval vectors $\hat{a}_{v}: \hat{a}_{v}=\left(\hat{a}_{1}, \hat{a}_{2}, \ldots, \hat{a}_{n}\right)^{T}, \hat{a}_{j} \in$ $I(\mathbb{R}), j=1,2, \ldots, n\}$.

- $\Lambda_{k}:\{1,2, \ldots, k\}$.

- An algebraic operation $\circledast(* \in\{+,-, \cdot, /\})$ in $I(\mathbb{R})$ is defined as follows. For $\hat{a}=\left[a^{L}, a^{R}\right]$ and $\hat{b}=\left[b^{L}, b^{R}\right]$ in $I(\mathbb{R}), \hat{a} \circledast \hat{b}=\{a * b: a \in \hat{a}, b \in \hat{b}\}$. Hence $\hat{a} \oplus \hat{b}=\left[a^{L}+b^{L}, a^{R}+b^{R}\right], \quad \hat{a} \ominus \hat{b}=\left[a^{L}-b^{R}, a^{R}-b^{L}\right]$, $\hat{a} \odot \hat{b}=\left[\min _{a \in \hat{a}, b \in \hat{b}} a . b, \max _{a \in \hat{a}, b \in \hat{b}} a . b\right]$ and $\hat{a} \oslash \hat{b}=\left[\min _{a \in \hat{a}, b \in \hat{b}} \frac{a}{b}, \max _{a \in \hat{a}, b \in \hat{b}} \frac{a}{b}\right], 0 \notin \hat{b}$.

- The spread of the interval $\hat{a}$ is $\mu(\hat{a})=a^{R}-a^{L}$.

- Summation and product of several intervals in $I(\mathbb{R})$ are denoted as follows. For $\hat{a}_{1}, \hat{a}_{2}, \ldots, \hat{a}_{n} \in I(\mathbb{R})$, $\widehat{\sum}_{j=1}^{n} \hat{a}_{j} \triangleq \hat{a}_{1} \oplus \hat{a}_{2} \oplus \ldots \oplus \hat{a}_{n}, \widehat{\prod}_{j=1}^{n} \hat{a}_{j} \triangleq \hat{a}_{1} \odot \hat{a}_{2} \odot \ldots \odot \hat{a}_{n}$.

\subsection{Interval valued function}

Interval valued function is defined by many authors in several ways (see [6,22], etc). In general, interval valued function is a mapping from one or more interval arguments onto an interval number. An interval valued function $\hat{f}: \mathbb{R}^{n} \rightarrow I(\mathbb{R})$ is $\hat{f}(x)=\left[f^{L}(x), f^{R}(x)\right]$ such that $f^{L}(x) \leq f^{R}(x) \forall x \in \mathbb{R}^{n}$.

\subsection{Order relations in $I(\mathbb{R})$}

The set of intervals is not a totally ordered set. Several partial orderings in $I(\mathbb{R})$ exist in literature(see $[1,6,22])$. Order relation between two intervals $\hat{a}$ and $\hat{b}$ can be explained in two ways; first one is an extension of $<$ on real line, that is, $\hat{a}<\hat{b}$ iff $a^{R}<b^{L}$, and the other is an extension of the concept of set inclusion, that is, $\hat{a} \subseteq \hat{b}$ iff $a^{L} \geq b^{L}$ and $a^{R} \leq b^{R}$. These order relations cannot explain ranking between two overlapping intervals. Set of intervals is a partial order set, so all intervals can not be compared with respect to a particular partial order relation. In most of the literatures on interval optimization, $\preceq_{L R}$ partial order relation is used to compare two intervals. According to this partial ordering, $\hat{a} \preceq_{L R} \hat{b}$ iff $a^{L} \leq b^{L}$ and $a^{R} \leq b^{R}$. But in case, when one interval lies in another one, then these two intervals are not comparable with respect to ${ }_{L R}$ partial order relation. For this reason we have introduced a new partial order relation, and named this as $\chi$ - partial order relation. Definition of $\chi$ - partial order relation and its advantage are provided in the following subsection. 


\subsection{1 $\chi$ - partial ordering}

Two intervals may overlap, one interval may lie behind another interval or one interval may contain another interval. To describe this concept mathematically, we associate a function $\chi: I(\mathbb{R}) \times I(\mathbb{R}) \rightarrow[0,1]$ as follows. For two intervals $\hat{a}$ and $\hat{b}$,

$$
\chi(\hat{a}, \hat{b})= \begin{cases}1, & a^{R} \leq b^{L}, \\ 0, \quad b^{R}-a^{L} & a^{L} \geq b^{R}, \\ \frac{\left(b^{R}-b^{L}\right)+\left(a^{R}-a^{L}\right)}{} \in(0,1), & a^{L}<b^{R} \text { and } a^{R}>b^{L} .\end{cases}
$$

$\chi(\hat{a}, \hat{b})$ represents degree of closeness of $\hat{a}$ with $\hat{b}$. One may observe here that $\chi$ is continuous and belongs to $[0,1]$. Moreover $\chi(\hat{a}, \hat{b})+\chi(\hat{b}, \hat{a})=1$.

We define order relation " $\chi_{\chi}$ " between two intervals as follows, which is based upon the concept of closeness of two intervals.

Definition 1. For two intervals $\hat{a}, \hat{b} \in I(\mathbb{R})$,

$$
\begin{aligned}
& \hat{a} \preceq_{\chi} \hat{b} \quad \text { iff } \mu(\hat{a}) \leq \mu(\hat{b}) \text { and } \chi(\hat{a}, \hat{b}) \in[1 / 2,1] \\
& \hat{a} \prec_{\chi} \hat{b} \quad \text { iff } \mu(\hat{a}) \leq \mu(\hat{b}) \text { and } \quad \chi(\hat{a}, \hat{b})=1 \\
& \hat{a}=\hat{b} \quad \text { iff } \mu(\hat{a})=\mu(\hat{b}) \text { and } \chi(\hat{a}, \hat{b})=1 / 2 .
\end{aligned}
$$

$\chi(\hat{a}, \hat{b}) \in\left[\frac{1}{2}, 1\right]$ means $\chi(\hat{a}, \hat{b}) \geq \chi(\hat{b}, \hat{a})$.

For example, $\mu([1,4])<\mu([0,5])$ and $\chi([1,4],[0,5])=\frac{1}{2}$. So $[1,4] \preceq \chi[0,5]$ with degree of closeness $\frac{1}{2}$;

$\chi([1,5],[3,6])=\frac{5}{7}$ but $[1,5] \preceq_{\chi}[3,6]$ is not true, so $\mu([1,5]) \not \leq \mu([3,6])$.

$\mu([1,4])<\mu([5,9])$ and $\chi([1,4],[5,9])=1$. So $[1,4] \prec_{\chi}[5,9]$ with degree of closeness 1 ;

$\mu([1,4])=\mu([-3,0])$ but $\chi([1,4],[-3,0])=0$, so $[1,4] \preceq_{\chi}[-3,0]$ is not true.

Lemma 1. $\preceq_{\chi}$ is a partial order relation.

Proof of this result is provided in Appendix.

Note 1. Advantages of $\chi$-partial ordering are the following:

$\chi$ - partial order relation describes the closeness between two intervals. According to this partial ordering, for any two intervals $\hat{a}$ and $\hat{b}$, closeness of $\hat{a}$ with $\hat{b}$ is $\chi(\hat{a}, \hat{b})$, which always lies between $50 \%$ to $100 \%$ and also known as degree of closeness. This means $\hat{a}$ is more than $50 \%$ closer towards $\hat{b}$. The existing interval partial orderings in the literature are either not associated with any such closeness factor or if associated, then their closeness/acceptability factor lies between $0 \%$ to $100 \%$.

Interval order relation due to [26] is associated with acceptability index, which lies between 0 and 1 . $\chi$ - partial ordering is associated with closeness index, which lies between $\frac{1}{2}$ and 1 . Both interval order relations determine closeness of one interval towards other. Since the degree of closeness between 
two intervals is always more than $50 \%$ in case of $\chi$-partial ordering, so $\chi$-partial ordering always accepts the decisions with higher degree of acceptability, which is definitely more acceptable for any decision maker. It is true that, the decision is more acceptable for the decision maker if the degree of closeness is more. Since our degree of closeness is always more then $50 \%$ so $\chi$ partial ordering is stronger than other partial ordering.

$I(\mathbb{R})^{n}$ is not a totally ordered set. To compare the interval vectors in $I(\mathbb{R})^{n}$, we define the following partial ordering $\preceq_{\chi}^{n}$.

Definition 2. For $\hat{a}_{v}=\left(\begin{array}{llll}\hat{a}_{1} & \hat{a}_{2} \ldots \hat{a}_{n}\end{array}\right)^{T}$ and $\hat{b}_{v}=\left(\begin{array}{llll}\hat{b}_{1} & \hat{b}_{2} & \ldots & \hat{b}_{n}\end{array}\right)^{T}$ in $I(\mathbb{R})^{n}$, $\hat{a}_{v} \preceq_{\chi}^{n} \hat{b}_{v} \quad$ iff $\hat{a}_{i} \preceq_{\chi} \hat{b}_{i}, i \in \Lambda_{n}$.

From the above two definitions, degree of closeness between two interval vectors $\hat{a}_{v}$ and $\hat{b}_{v}$ of dimension $n$ can be defined as follows.

Definition 3. Degree of closeness of the interval vector $\hat{a}_{v}=\left(\begin{array}{llll}\hat{a}_{1} & \hat{a}_{2} & \ldots & \hat{a}_{n}\end{array}\right)^{T}$ with the interval vector $\hat{b}_{v}=\left(\hat{b}_{1} \hat{b}_{2} \ldots \hat{b}_{n}\right)^{T}$ is defined as

$$
\chi\left(\hat{a}_{v}, \hat{b}_{v}\right)=\min _{i \in \Lambda_{n}}\left\{\chi\left(\hat{a}_{i}, \hat{b}_{i}\right)\right\} .
$$

Example 1. Consider the interval vectors $\hat{a}_{v}=([0,2],[2,3])^{\mathbf{T}}$ and $\hat{b}_{v}=([1,4]$, $[2,4])^{\mathbf{T}}$. Here $\chi([0,2],[1,4])=\frac{4}{5}, \chi([2,3],[2,4])=\frac{2}{3}, \chi\left(\hat{a}_{v}, \hat{b}_{v}\right)=\min \{\chi([0,2]$, $[1,4]), \chi([2,3],[2,4])\}=\frac{2}{3}$. Hence we say $\hat{a}_{v} \preceq_{\chi}^{2} \hat{b}_{v}$ with degree of closeness $\frac{2}{3}$.

Throughout this paper we consider the partial ordered sets $\left(I(\mathbb{R}), \preceq_{\chi}\right)$ and $\left(I(\mathbb{R})^{n}, \preceq_{\chi}^{n}\right)$.

\section{Interval multi-objective geometric programming prob- lem and its solution}

In a general multi-objective optimization problem there may not exist a single optimal solution that simultaneously optimizes all the objective functions. In this circumstance the decision maker looks for the most preferred solution. Hence the concept of optimal solution is replaced with Pareto-optimal/ efficient solution. This concept may be extended for multi-objective geometric programming problem with interval parameters.

Consider a general interval multi-objective geometric programming problem $(I M G P)$ with $k$ objective functions as,

$(I M G P):$

$$
\begin{array}{r}
\min \left\{\widehat{\sum}_{t_{1}=1}^{s_{01}} \hat{c}_{0 t_{1}} \widehat{\prod}_{l=1}^{n} x_{l}^{\hat{\gamma}_{0 l 1}}, \widehat{\sum}_{t_{2}=1}^{s_{02}} \hat{c}_{0 t_{2}} \widehat{\prod}_{l=1}^{n} x_{l}^{\hat{\gamma}_{0 l 2}}, \ldots,\right. \\
\left.\widehat{\sum}_{t_{k}=1}^{s_{0 k}} \hat{c}_{0 t_{k}} \widehat{\prod}_{l=1}^{n} x_{l}^{\hat{\gamma}_{0 l k}}\right\}
\end{array}
$$

subject to $\quad \widehat{\sum}_{t=1}^{s_{j}} \hat{c}_{j t} \widehat{\prod}_{l=1}^{n} x_{l}^{\hat{\gamma}_{j t l}} \preceq \hat{b}_{j}, j \in \Lambda_{m}, \quad x>0$, 
where $\hat{c}_{0 t_{i}}, \hat{\gamma}_{0 l i}, \hat{c}_{j t}, \hat{\gamma}_{j t l}, \quad \hat{b}_{j} \in I(\mathbb{R})_{+}, \hat{c}_{0 t_{i}}=\left[c_{0 t_{i}}^{L}, c_{0 t_{i}}^{R}\right], \hat{\gamma}_{0 l i}=\left[\gamma_{0 l i}^{L}, \gamma_{0 l i}^{R}\right]$, $\hat{c}_{j t}=\left[c_{j t}^{L}, c_{j t}^{R}\right], \hat{b}_{j}=\left[b_{j}^{L}, b_{j}^{R}\right]$ with $c_{0 t_{i}}^{L}>0, c_{j t}^{L}>0$ and $b_{j}^{L}>0, \forall t_{i}, j, t, i \in \Lambda_{k}$, $j \in \Lambda_{m}$.

Solution method for $(I M G P)$ is different from the solution method for general multi objective geometric programming problem due the presence of interval uncertainties and interval ordering, associated with $(I M G P)$. Solution of $(I M G P)$ is definitely a compromising solution but following uncertainties should be taken care.

(i) Feasible region of $(I M G P)$ is the set

$$
\left\{x:{\widehat{\sum_{t=1}^{s_{j}}}}_{\hat{c}_{j t}} \widehat{\prod}_{l=1}^{n} x_{l}^{\hat{\gamma}_{j t l}} \preceq \hat{b}_{j}, \quad \forall j \in \Lambda_{m}\right\},
$$

which has uncertain parameters as intervals as well as interval ordering. So $x \in \mathbb{R}^{n}$ can be a feasible solution of $(I M G P)$ if $x$ satisfies $m$ number of interval inequalities, $\widehat{\sum}_{t=1}^{s_{j}} \hat{c}_{j t} \widehat{\prod}_{l=1}^{n} x_{l}^{\hat{\gamma}_{j t l}} \preceq{\hat{b_{j}}}_{j}$, which can be determined using the concept of closeness between two interval vectors as per Definition 3. Hence a feasible point is associated with certain degree of closeness between two interval vectors. In other words, we may say this feasible point with some degree of closeness as a feasible solution with some acceptability level.

(ii) A feasible point $x$ with certain degree of closeness/acceptability level, can be an efficient solution of (IMGP) for the $k$ conflicting interval valued objective functions. This has another uncertain factor in connection to these $k$ conflicting interval functions, which are compared using partial ordering $\left(\preceq_{\chi}^{k}\right)$. We say, any acceptable feasible solution which optimizes all these conflicting objectives functions, as $\chi$-efficient solution.

These two uncertainties are addressed separately in mathematical terms in the following subsections to find an efficient solution of $(I M G P)$.

\subsection{Acceptable feasible solution}

The feasible region of $(I M G P)$ is the set

$$
S=\left\{x \in \mathbb{R}^{n}: \widehat{\sum}_{t=1}^{s_{j}} \hat{c}_{j t} \widehat{\prod}_{l=1}^{n} x_{l}^{\hat{\gamma}_{j t l}} \preceq \hat{b}_{j}, j \in \Lambda_{m}\right\} .
$$

$S$ is associated with a system of $m$ non-linear inequalities. Acceptable feasible solution for $(I M G P)$ can be derived in the light of the discussion on closeness between two interval vectors $\left(\widehat{\sum}_{t=1}^{s_{1}} \hat{c}_{1 t} \widehat{\prod}_{l=1}^{n} x_{l}^{\hat{\gamma}_{1 t l}} \widehat{\sum}_{t=1}^{s_{2}} \hat{c}_{2 t} \widehat{\prod}_{l=1}^{n} x_{l}^{\hat{\gamma}_{2 t l}} \ldots\right.$ \left.${\widehat{\sum_{t=1}^{s_{m}}}}_{\hat{c}_{m t}} \widehat{\prod}_{l=1}^{n} x_{l}^{\hat{\gamma}_{m t l}}\right)^{\mathbf{T}}$ and $\left(\hat{b_{1}} \hat{b_{2}} \cdots \hat{b_{m}}\right)^{\mathbf{T}}$ (See Definition 3). Here, for every $x \in \mathbb{R}^{n}$,

$$
{\widehat{\sum_{t=1}}}_{s_{j}} \hat{c}_{j t} \widehat{\prod}_{l=1}^{n} x_{l}^{\hat{\gamma}_{j t l}}=\left[\sum_{t=1}^{s_{j}} c_{j t}^{L} \prod_{l=1}^{n} x_{l}^{\eta_{j t l}}, \sum_{t=1}^{s_{j}} c_{j t}^{R} \prod_{l=1}^{n} x_{l}^{\eta_{j t l}}\right],
$$


where

$$
x_{l}^{\eta_{j t l}}= \begin{cases}x_{l}^{\gamma_{j t l}^{L}}, & \text { when } x_{l} \geq 1, \\ x_{l}^{\gamma_{j t l}^{R},}, & \text { when } x_{l}<1 .\end{cases}
$$

For any $x$ in $S$, the interval $\left[\sum_{t=1}^{s_{j}} c_{j t}^{L} \prod_{l=1}^{n} x_{l}^{\eta_{j t l}}, \sum_{t=1}^{s_{j}} c_{j t}^{R} \prod_{l=1}^{n} x_{l}^{\eta_{j t l}}\right]$ may lie behind or overlap or exceed $\left[b_{j}^{L}, b_{j}^{R}\right]$ for every $j \in \Lambda_{m}$. Accordingly the feasibility of $x$ for $(I M G P)$ is completely acceptable or partially acceptable or not acceptable. Hence every point $x$ in $S$ is associated with certain degree of acceptability/feasibility/closeness factor. Using the discussion in Section 2, we will convert $S$ to a deterministic form to have some mathematical sense of this affect as follows.

Denote

$$
S_{\text {max }}=\left\{x: \sum_{t=1}^{s_{j}} c_{j t}^{L} \prod_{l=1}^{n} x_{l}^{\eta_{j t l}} \leq b_{j}^{R} j \in \Lambda_{m}\right\}
$$

and

$$
S_{\text {min }}=\left\{x: \sum_{t=1}^{s_{j}} c_{j t}^{R} \prod_{l=1}^{n} x_{l}^{\eta_{j t l}} \leq b_{j}^{L} \quad j \in \Lambda_{m}\right\} .
$$

$S_{\max }$ and $S_{\min }$ are the maximum and minimum feasible regions respectively. From the definition of $S_{\max }$ and $S_{\min }$, it is obvious that $S_{\min } \subseteq S_{\max }$. Hence any feasible point of $(I M G P)$ lies either in $S_{\min }$ or in $S_{\max } \backslash S_{\min }$, but not in the complement of $S_{\max }$ (which is $S_{\max }^{c}$ ), depending upon the relation between $\left[\sum_{t=1}^{s_{j}} c_{j t}^{L} \prod_{l=1}^{n} x_{l}^{\eta_{j t l}}, \sum_{t=1}^{s_{j}} c_{j t}^{R} \prod_{l=1}^{n} x_{l}^{\eta_{j t l}}\right]$ and $\left[b_{j}^{L}, b_{j}^{R}\right]$. It is clear that

(i) $x \in S$ is a fully acceptable feasible solution if $x \in S_{\min }$. i.e.,

$$
\sum_{t=1}^{s_{j}} c_{j t}^{R} \prod_{l=1}^{n} x_{l}^{\eta_{j t l}} \leq b_{j}^{L} \quad j \in \Lambda_{m} ;
$$

(ii) $x$ is not at all an acceptable feasible point if $x$ goes beyond the region $S_{\max }$. i.e.,

$$
x \in S_{\max }^{c}, \sum_{t=1}^{s_{j}} c_{j t}^{L} \prod_{l=1}^{n} x_{l}^{\eta_{j t l}}>b_{j}^{R} j \in \Lambda_{m} ;
$$

(iii) $x$ is a partially acceptable feasible solution if $x \in S_{\max } \backslash S_{\min }$. In this case, the degree of acceptability of $x$ decreases from $100 \%$ to $0 \%$ as it moves closer to $S_{\max }$ from $S_{\min }$.

Acceptability degree of $x$ corresponding to $j^{\text {th }}$ constraint is the degree of closeness of two intervals $\widehat{\sum}_{t=1}^{s_{j}} \hat{c}_{j t} \widehat{\prod}_{l=1}^{n} x_{l}^{\hat{\gamma}_{j t l}}$ and $\hat{b}_{j}$, which can be obtained by associating a function $\chi_{j}^{F}: I(\mathbb{R}) \times I(\mathbb{R}) \rightarrow[0,1]$ as in $(2.1)$, as follows.

$$
\begin{aligned}
\chi_{j}^{F}\left(\widehat{\sum}_{t=1}^{s_{j}} \hat{c}_{j t} \widehat{\prod}_{l=1}^{n} x_{l}^{\hat{\gamma}_{j t l}}, \hat{b}_{j}\right) \\
\quad= \begin{cases}1, & \sum_{t=1}^{s_{j}} c_{j t}^{R} \prod_{l=1}^{n} x_{l}^{\eta_{j t l}} \leq b_{j}^{L}, \\
0, & \sum_{t=1}^{s_{j}} c_{j t}^{L} \prod_{l=1}^{n} x_{l}^{\eta_{j t l}}>b_{j}^{R}, \\
\frac{b_{j}^{R}-\sum_{t=1}^{s_{j}} c_{j t}^{L} \prod_{l=1}^{n} x_{l}^{\eta_{j t l}}}{\left(b_{j}^{R}-b_{j}^{L}\right)+\left(\sum_{t=1}^{s_{j}} c_{j t}^{R} \prod_{l=1}^{n} x_{l}^{\eta_{j t l}}-\sum_{t=1}^{s_{j}} c_{j t}^{L} \prod_{l=1}^{n} x_{l}^{\eta_{j t l}}\right)}, & \text { elsewhere. }\end{cases}
\end{aligned}
$$


It is clear that every $x \in S$ is associated with certain degree of acceptability $\left(\chi_{j}^{F}\right)$ with respect to $j^{\text {th }}$ constraint. Since $S$ is the intersection of $m$ number of constraints, so every $x \in S$ satisfies the minimum degree of closeness/acceptablity, which can be found using Definition 3 as

$$
\tau=\min _{1 \leq j \leq m}\left\{\chi_{j}^{F}\left(\widehat{\sum}_{t=1}^{s_{j}} \hat{c}_{j t} \widehat{\prod}_{l=1}^{n} x_{l}^{\hat{\gamma}_{j t l}}, \hat{b}_{j}\right)\right\} .
$$

Define a set

$$
\mathbf{S}^{\prime}=\left\{(x, \tau): x \in \mathbb{R}^{n}, \tau=\min _{1 \leq j \leq m}\left\{\chi_{j}^{F}\left(\widehat{\sum}_{t=1}^{s_{j}} \hat{c}_{j t} \widehat{\prod}_{l=1}^{n} x_{l}^{\hat{\gamma}_{j t l}}, \hat{b}_{j}\right)\right\}\right\} .
$$

For $(x, \tau) \in \mathbf{S}^{\prime}$, we say $x$ is a feasible point with acceptable degree $\tau$ and $\mathbf{S}^{\prime}$ is the acceptable feasible region.

\section{2 $\chi$-efficient solution}

Since $(I M G P)$ is a multi-objective programming problem so it may not have optimal solution as in the case of a single objective optimization problem. So it is necessary to determine Pareto-optimal/compromising/efficient solution of $(I M G P)$ over this acceptable feasible region $\mathbf{S}^{\prime}$. In other words we need to solve

$$
\begin{array}{r}
\min _{(x, \tau) \in \mathbf{S}^{\prime}}\left\{\widehat{\sum}_{t_{1}=1}^{s_{01}} \hat{c}_{0 t_{1}} \widehat{\prod}_{l=1}^{n} x_{l}^{\hat{\gamma}_{0 l 1}},\right. \\
\widehat{\sum}_{t_{2}=1}^{s_{02}} \hat{c}_{0 t_{2}} \widehat{\prod}_{l=1}^{n} x_{l}^{\hat{\gamma}_{0 l 2}}, \ldots, \\
\left.\widehat{\sum}_{t_{k}=1}^{s_{0 k}} \hat{c}_{0 t_{k}} \widehat{\prod}_{l=1}^{n} x_{l}^{\hat{\gamma}_{0 l k}}\right\} .
\end{array}
$$

Recall that a feasible solution of a general multi-objective programming problem is an efficient solution if there is no other feasible solution that would reduce some objective value without causing simultaneous increase in at least one other objective value. This type situation appears in an interval multi-objective geometric programming (IMGP) also. An exact optimum solution of an interval multi-objective geometric programming problem may not be found always due to the nature of conflicting objectives. Hence the decision maker has to compromise with several objective values. Here each objective value is an interval, which leads to uncertainty. For this purpose partial orderings are necessary to compare interval vectors as well as intervals in place of real vectors and real numbers respectively. To compare interval valued objective functions in (IMGP), we accept $\preceq_{\chi}$ and $\preceq_{\chi}^{n}$ partial orderings as discussed in Section 2.2.1.

In the light of the definition of weak efficient solution of a general multiobjective geometric programming problem $(M G P)$, we define weak efficient solution of $(I M G P)$ with respect to $\preceq_{\chi}^{k}$ partial ordering in $I(\mathbb{R})^{k}$ and call this solution as $\chi$-efficient solution.

Definition 4. A feasible solution $(x, \tau)$ with acceptable degree $\tau$ of $(I M G P)$ is said to be $\chi$-efficient solution of (IMGP) if there does not exist any feasible solution $\left(y, \tau^{\prime}\right)$ with acceptable degree $\tau^{\prime},\left(\tau^{\prime} \geq \tau\right)$, of $(I M G P)$ such that

$$
\widehat{\sum}_{t_{i}=1}^{s_{0 i}} \hat{c}_{0 t_{i}} \widehat{\prod}_{l=1}^{n} y_{l}^{\hat{\gamma}_{0 l i}} \prec \chi \widehat{\sum}_{t_{i}=1}^{s_{0 i}} \hat{c}_{0 t_{i}} \widehat{\prod}_{l=1}^{n} x_{l}^{\hat{\gamma}_{0 l i}} \forall i \in \Lambda_{k} .
$$


Note that $\tau^{\prime} \geq \tau$ is considered since $\tau^{\prime}<\tau$ implies that $y$ has less feasibility degree, which can not be acceptable for a decision maker.

\subsubsection{Solution of $(P)$}

It is difficult to derive the $\chi$-efficient solution (Definition 4) of (IMGP) analytically. For this purpose we assign a target/goal to every objective function of $(I M G P)$. Since every objective function is an interval valued function, so the decision maker has an aspiration level of achievement (denoted by $l_{i}$ ) and highest possible level of achievement (denoted by $u_{i}$ ) of $i^{\text {th }}$ objective function for every $i .\left[l_{i}, u_{i}\right]$ can be considered as preassigned goal for $i^{\text {th }}$ objective function. The goal $\left[l_{i}, u_{i}\right]$ stands for the achievement level of the objective function which is to be minimized. That is, the decision maker has to take the decisions so that

$$
\widehat{\sum}_{t_{i}=1}^{s_{0 i}} \hat{c}_{0 t_{i}} \widehat{\prod}_{l=1}^{n} x_{l}^{\hat{\gamma}_{0 l i}} \preceq_{\chi}\left[l_{i}, u_{i}\right] \forall i
$$

where

$$
{\widehat{\sum_{t_{i}=1}}}_{s_{0 i}}^{s_{0 t_{i}}} \widehat{\prod}_{l=1}^{n} x_{l}^{\hat{\gamma}_{0 l i}}=\left[\sum_{t_{i}=1}^{s_{0 i}} c_{0 t_{i}}^{L} \prod_{l=1}^{n} x_{l}^{\eta_{0 l i}}, \sum_{t_{i}=1}^{s_{0 i}} c_{0 t_{i}}^{R} \prod_{l=1}^{n} x_{l}^{\eta_{0 l i}}\right] .
$$

Value of these goals $\left(l_{i}\right.$ and $\left.u_{i}\right)$ may be provided by the decision maker. The basic idea behind this assumption is that, the decision maker specifies desired goal levels for the objective functions, and the actual optimization problem uses the deviations from the goals as the objective of the model. $l_{i}$ is aspire level of achievement and $u_{i}$ is the highest acceptable level of achievement of the objective function. This means, minimum value of $i^{t h}$ objective function is very close to $\left[l_{i}, u_{i}\right]$.

The inequalities (3.3) literally mean that, for every $(x, \tau) \in \mathbf{S}^{\prime}$, deviation of $i^{\text {th }}$ objective function from the goal $\left[l_{i}, u_{i}\right]$ may be more or less acceptable for the decision maker. Hence every interval valued objective function is associated with certain degree of flexibility from its goal. One may observe that for every $(x, \tau) \in \mathbf{S}^{\prime}$, the degree of flexibility of $\widehat{\sum}_{t_{i}=1}^{s_{0 i}} \hat{c}_{0 t_{i}} \widehat{\prod}_{l=1}^{n} x_{l}^{\hat{\gamma}_{0 l i}}$ is higher if deviation of $\sum_{t_{i}=1}^{s_{0 i}} c_{0 t_{i}}^{R} \prod_{l=1}^{n} x_{l}^{\eta_{0 l i}}$ from $l_{i}$ is less and the degree of flexibility is less if deviation of $\sum_{t_{i}=1}^{s_{0 i}} c_{0 t_{i}}^{R} \prod_{l=1}^{n} x_{l}^{\eta_{0 l i}}$ from $u_{i}$ is more. This logic is similar to the discussion of Subsection 2.2.1 in the context of the closeness between two intervals. Accordingly we can associate a function $\chi_{j}^{O}: I(\mathbb{R}) \times I(\mathbb{R}) \rightarrow[0,1]$ as in (2.1) to measure this degree of closeness, as

$$
\begin{aligned}
\chi_{i}^{O}\left(\widehat{\sum}_{t_{i}=1}^{s_{0 i}} \hat{c}_{0 t_{i}} \widehat{\prod}_{l=1}^{n} x_{l}^{\hat{\gamma}_{0 l i}}, \quad\left[l_{i}, u_{i}\right]\right) \\
\quad= \begin{cases}1, & \text { if } \sum_{t_{i}=1}^{s_{0 i}} c_{0 t_{i}}^{R} \prod_{l=1}^{n} x_{l}^{\eta_{0 l i}} \leq l_{i}, \\
0, & \text { if } \sum_{t_{i}=1}^{s_{0 i}} c_{0 t_{i}}^{L} \prod_{l=1}^{n} x_{l}^{\eta_{0 l i}} \geq u_{i}, \\
\frac{u_{i}-\sum_{t_{i}=1}^{s_{0}} c_{0 t_{i}}^{L} \prod_{l=1}^{n} x_{l}^{\eta_{0 l i}}}{\left(u_{i}-l_{i}\right)+\left(\sum_{t_{i}=1}^{s_{0 i}} c_{0 t_{i}}^{R} \prod_{l=1}^{n} x_{l}^{\eta_{0 l i}}-\sum_{t_{i}=1}^{s_{0 i}} c_{0 t_{i}}^{L} \prod_{l=1}^{n} x_{l}^{\eta_{0 l i}}\right)}, \text { elsewhere. }\end{cases}
\end{aligned}
$$

Here, value of every objective function is flexible towards its goal with certain 
degree of flexibility. This occurs simultaneously for $k$ number of goals with minimum flexibility, $\min _{i} \chi_{i}^{O}\left(\widehat{\sum}_{t_{i}=1}^{s_{0 i}} \hat{c}_{0 t_{i}} \widehat{\prod}_{l=1}^{n} x_{l}^{\hat{\gamma}_{0 l i}},\left[l_{i}, u_{i}\right]\right)$ (See Definition 3).

The objective functions are characterized by their individual degree of flexibility and the constraints are characterized by their degree of acceptability. So, in this uncertain environment a decision $x$ is the selection of activities that simultaneously satisfy all the objective functions and constraints with maximum satisfaction, which may be mathematically expressed as

$$
\begin{aligned}
& \max \min _{i}\left\{\chi_{i}^{O}\left(\widehat{\sum}_{t_{i}=1}^{s_{0 i}} \hat{c}_{0 t_{i}} \widehat{\prod}_{l=1}^{n} x_{l}^{\hat{\gamma}_{0 l i}},\left[l_{i}, u_{i}\right]\right) ;(x, \tau) \in \mathbf{S}^{\prime}\right\} \\
& \equiv \max _{i} \min _{i}\left\{\chi_{i}^{O}\left(\widehat{\sum}_{t_{i}=1}^{s_{0 i}} \hat{c}_{0 t_{i}} \widehat{\prod}_{l=1}^{n} x_{l}^{\hat{\gamma}_{0 l i}},\left[l_{i}, u_{i}\right]\right) ;\right. \\
& \left.\tau=\min _{j} \chi_{j}^{F}\left(\widehat{\sum}_{t=1}^{s_{j}} \hat{c}_{j t} \widehat{\prod}_{l=1}^{n} x_{l}^{\hat{\gamma}_{j t l}}, \hat{b}_{j}\right), x \in S\right\} .
\end{aligned}
$$

This max-min problem is equivalent to

$$
\begin{array}{ll}
(I M G P)^{\prime}: \max & \theta \\
\text { subject to } & \theta \leq \chi_{i}^{O}\left(\widehat{\sum}_{t_{i}=1}^{s_{0 i}} \hat{c}_{0 t_{i}} \widehat{\prod}_{l=1}^{n} x_{l}^{\hat{\gamma}_{0 l i}},\left[l_{i}, u_{i}\right]\right), i \in \Lambda_{k}, \\
& \theta \leq \chi_{j}^{F}\left(\widehat{\sum}_{t=1}^{s_{j}} \hat{c}_{j t} \widehat{\prod}_{l=1}^{n} x_{l}^{\hat{\gamma}_{j t l}}, \hat{b}_{j}\right), j \in \Lambda_{m}, 0<\theta<1 .
\end{array}
$$

After substituting the value of $\chi_{i}^{O}$ and $\chi_{j}^{F},(I M G P)^{\prime}$ can be further simplified to the following form.

$$
\begin{aligned}
& (I M G P)^{\prime}: \quad \max \theta \\
& \text { subject to }\left(\sum_{t_{i}=1}^{s_{0 i}} \theta c_{0 t_{i}}^{R} \prod_{l=1}^{n} x_{l}^{\eta_{0 l i}}\right)+\left(\sum_{t_{i}=1}^{s_{0 i}}(1-\theta) c_{0 t_{i}}^{L} \prod_{l=1}^{n} x_{l}^{\eta_{0 l i}}\right) \leq l_{i} \theta+(1-\theta) u_{i}, \\
& \quad\left(\sum_{t=1}^{s_{j}} \theta c_{j t}^{R} \prod_{l=1}^{n} x_{l}^{\eta_{j t l}}\right)+\left(\sum_{t=1}^{s_{j}}(1-\theta) c_{j t}^{L} \prod_{l=1}^{n} x_{l}^{\eta_{j t l}}\right) \leq b_{j}^{L} \theta+(1-\theta) b_{j}^{R}, \\
& \frac{1}{2}<\theta<1, i \in \Lambda_{k}, j \in \Lambda_{m} .
\end{aligned}
$$

$(I M G P)^{\prime}$ is a general geometric programming problem which is free from interval uncertainty, and can be solved using geometric programming technique. Let the solution of the problem $(I M G P)^{\prime}$ be $\left(\theta^{o p t}, x^{o p t}\right)$ with degree of feasibility $\tau^{o p t}$. Following result establishes the relation between the solution of $(I M G P)^{\prime}$ and $(I M G P)$.

Theorem 1. If $\left(\theta^{\text {opt }}, x^{\text {opt }}\right)$ is an optimal solution of the $(I M G P)^{\prime}$, then $x^{\text {opt }}$ is an $\chi$-efficient solution of (IMGP) with degree of satisfaction $\theta^{\text {opt }}$. In case of alternate optimal solution of $(I M G P)^{\prime}$, at least one of them is an $\chi$-efficient solution of $(I M G P)$.

Proof of the theorem is provided in Appendix.

Methodology of this section is illustrated in a waste water treatment model in next section. 


\section{A possible application in waste water treatment system}

Several problems in waste water treatment system can be formulated as an optimization model. Readers may refer [4,5,14,19,21,24,25,27,29] for different type of optimization models related to waste water treatment system. These are single objective optimization models where the treatment cost is minimized under several conditions while removing the pollutant in the water. One may observe that the total time required to execute the complete process of waste water treatment system plays an important role while minimizing the total cost. In this paper we address both the objectives which occur simultaneously: minimization of the total annual cost as well as total time required to complete all the steps of a waste water treatment plant. At every step, cost and time may vary due to the presence of several inexact information in the system, which arise due to change in climate, change in market price, quality of ingredients and instruments, which are used in the treatment process. Lower and upper bound of these parameters can be estimated from the historical data. As a result of which, the parameters of the waste water treatment model become intervals and hence the model is converted to interval optimization model. Formulation of such a model is discussed in detail in this section.

\subsection{System description}

A general waste water treatment system is configured in the following major steps.

Step 0 A known quantity of water from showers, toilets, washers and sometimes from factories is pumped into the tank where the water is dozed with alum for coagulation with heavy metals or insoluble particles. After coagulation, water is allowed to settle for some hours in the tanks.

Step I Next, water pollutants are removed by reverse osmosis process in which water is pushed through a semi-permeable membrane to remove salts, viruses etc. Then, the rest amount of water is taken to chlorination tank where the primary disinfection is brought about by bubbling chlorine gas.

Step II Water in Step I is then passed through sand filters for trapping of undissolved pollutants and also pass through carbon filters to remove odor, color etc. Dechlorination is done at this stage.

Step III Water from Step II is passed through a series of micro filters to remove bacteria, protozoa etc., followed by ultraviolet disinfection system for terminal disinfection.

Finally, water is packed in bottles through an automatic rising, filling and capping machine fitted with an ozone generator.

Let $x_{j}$ (in percentage) be the remaining amount of water pollutant after completion of $j^{\text {th }}$ step. Due to change in climate, change in market price, quality of ingredients and instruments used in the treatment process, total 
expenditure at $j^{\text {th }}$ step varies between $y_{j}^{L}$ and $y_{j}^{R}$ (say); and total time required to complete this step varies between $t_{j}^{L}$ and $t_{j}^{R}$ (say). Denote $\hat{y}_{j}\left(x_{j}\right)=$ $\left[y_{j}^{L}, y_{j}^{R}\right], \hat{t}_{j}\left(x_{j}\right)=\left[t_{j}^{L}, t_{j}^{R}\right]$, for $j=0,1,2,3$.

The required time and cost at every step depend upon the remaining amount of pollutants of that step. Objective of the system is to minimize the total cost and the total time required for the waste water treatment process so that at least $p \%$ (say) of the pollutants should be removed. The intervals $\hat{y}_{j}\left(x_{j}\right)$ and $\hat{t}_{j}\left(x_{j}\right)$ can be determined from some given past data.

\subsection{Formulation of the model}

To illustrate the proposed model, consider the following hypothetical data. Cost and time required for $l^{\text {th }}(l=1,2, \ldots, 6)$ experiment are given in Table 1 and Table 2 respectively. $x_{j l}$ (with percentage) denotes the remaining amount of pollutant after completion of step $j$. Cost and required time to complete $j^{\text {th }}$ step in $l^{t h}$ experiment varies in the intervals $\hat{y}_{j}\left(x_{j}\right)$ and $\hat{t}_{j}\left(x_{j}\right)$ respectively.

The intervals $\hat{y}_{j}\left(x_{j}\right)$ and $\hat{t}_{j}\left(x_{j}\right)$ at $j^{\text {th }}$ step of the model can be found from

Table 1. Cost to complete the steps for System.

\begin{tabular}{clccccc}
\hline & $\begin{array}{c}\text { Exp-1 } \\
(l=1)\end{array}$ & $\begin{array}{c}\text { Exp-2 } \\
(l=2)\end{array}$ & $\begin{array}{c}\text { Exp-3 } \\
(l=3)\end{array}$ & $\begin{array}{c}\text { Exp-4 } \\
(l=4)\end{array}$ & $\begin{array}{c}\text { Exp-5 } \\
(l=5)\end{array}$ & $\begin{array}{c}\text { Exp-6 } \\
(l=6)\end{array}$ \\
\hline$x_{j l} \rightarrow$ & $50 \%$ & $30 \%$ & $20 \%$ & $15 \%$ & $10 \%$ & $3 \%$ \\
$\hat{y}_{j l}=\left[y_{j l}^{L}, y_{j l}^{R}\right] \downarrow$ & & & & & & \\
\hline Step 0 $(j=0)$ & $\times$ & $\times$ & $\times$ & $\times$ & $\times$ & $\times$ \\
Step I $(j=1)$ & {$[0.5,0.8]$} & {$[1,1.2]$} & {$[1.4,1.5]$} & {$[1.5,1.8]$} & {$[2,2.5]$} & {$[3,3.5]$} \\
Step II $(j=2)$ & {$[0.5,0.6]$} & {$[0.7,0.9]$} & {$[2.5,2.7]$} & {$[2.8,3.1]$} & {$[3.3,3.5]$} & {$[3.8,4.2]$} \\
Step III $(j=3)$ & {$[1.4,1.5]$} & {$[1.8,2.1]$} & {$[2.8,3.1]$} & {$[3.2,3.5]$} & {$[3.8,4.0]$} & {$[4.4,4.6]$} \\
\hline
\end{tabular}

Table 2. Time taken to complete the steps for System.

\begin{tabular}{clccccc}
\hline & $\begin{array}{c}\text { Exp-1 } \\
(l=1)\end{array}$ & $\begin{array}{c}\text { Exp-2 } \\
(l=2)\end{array}$ & $\begin{array}{c}\text { Exp-3 } \\
(l=3)\end{array}$ & $\begin{array}{c}\text { Exp-4 } \\
(l=4)\end{array}$ & $\begin{array}{c}\text { Exp-5 } \\
(l=5)\end{array}$ & $\begin{array}{c}\text { Exp-6 } \\
(l=6)\end{array}$ \\
\hline $\begin{array}{c}x_{j l} \rightarrow \\
\hat{t}_{j l}=\left[t_{j l}^{L}, t_{j l}^{R}\right] \downarrow\end{array}$ & $50 \%$ & $30 \%$ & $25 \%$ & $15 \%$ & $10 \%$ & $5 \%$ \\
\hline Step 0 $(j=0)$ & $\times$ & $\times$ & $\times$ & $\times$ & $\times$ & $\times$ \\
Step I $(j=1)$ & {$[0.8,1.0]$} & {$[1,1.3]$} & {$[1.5,1.7]$} & {$[1.8,2.0]$} & {$[2.1,2.4]$} & {$[3.0,3.2]$} \\
Step II $(j=2)$ & {$[0.4,0.5]$} & {$[0.6,0.8]$} & {$[2.4,2.5]$} & {$[2.6,2.8]$} & {$[3.2,3.5]$} & {$[3.6,3.9]$} \\
Step III $(j=3)$ & {$[1.4,1.6]$} & {$[2.8,2.9]$} & {$[2.9,3.2]$} & {$[3.4,3.6]$} & {$[4.1,4.3]$} & {$[4.4,4.6]$} \\
\hline
\end{tabular}

this data through interpolation. Here we consider least square approximation to interpolate data as follows.

For $j=0,1,2,3$ and $a_{j}, b_{j} \in \mathbb{R}, b_{j}>0$, consider the interpolating curve $y_{j l}=b_{j} x_{j l} a_{j}$, where $y_{j l} \in \hat{y}_{j l}$. Then $y_{j l}^{\prime}=b_{j}^{\prime}+a_{j} x_{j l}^{\prime}$, where $y_{j l}^{\prime}=\log y_{j l}, b_{j}^{\prime}=$ 
$\log b_{j}, x_{j l}^{\prime}=\log x_{j l}$. For best approximation, $b_{j}^{\prime}$ and $a_{j}$ should be found in such a way that $\sum_{l=1}^{6}\left(a_{j} x_{j l}^{\prime}+b_{j}^{\prime}-y_{j l}^{\prime}\right)^{2}$ is minimum. The necessary and sufficient condition for the existence of the minimum of $L$, where $L=\sum_{l=1}^{6}\left(a_{j} x_{j l}^{\prime}+b_{j}^{\prime}-y_{j l}^{\prime}\right)^{2}$, are equations $\frac{\partial L}{\partial a_{j}}=0$ and $\frac{\partial L}{\partial b_{j}^{\prime}}=0$. Solving this system, we get

$$
\begin{aligned}
& a_{j}=\frac{6 \sum_{l=1}^{6} \log x_{j l} \log y_{j l}-\sum_{l=1}^{6} \log x_{j l} \sum_{l=1}^{6} \log y_{j l}}{6 \sum_{l=1}^{6}\left(\log x_{j l}\right)^{2}-\left(\sum_{l=1}^{6} \log x_{j l}\right)^{2}}, \\
& b_{j}^{\prime}=\frac{1}{6}\left(\sum_{l=1}^{6} \log y_{j l}-a_{j} \sum_{l=1}^{6} \log x_{j l}\right),
\end{aligned}
$$

$b_{j}$ can be found from the relation $b_{j}^{\prime}=\log b_{j}$. Since $y_{j l} \in \hat{y}_{j l}$ so $a_{j}$ and $b_{j}$ also lie in some intervals, which can be computed as follows

$$
a_{j} \in\left[\min _{y_{j l} \in \hat{y}_{j l}} a_{j}, \max _{y_{j l} \in \hat{y}_{j l}} a_{j}\right]=\hat{a}_{j} \text { (say), } b_{j} \in\left[\min _{y_{j l} \in \hat{y}_{j l}} b_{j}, \max _{y_{j l} \in \hat{y}_{j l}} b_{j}\right]=\hat{b}_{j} \text { (say). }
$$

Hence the cost function at $j^{t h}$ step is $\hat{y}_{j}=\hat{b}_{j} \odot x_{j}{ }^{\hat{a}_{j}}$, which is an interval valued function, and can be interpolated from the data given in Table 1. Similarly the time function at $j^{\text {th }}$ can be calculated from the data given in Table 2 through an interpolating function $t_{j}=d_{j} x_{j}^{c_{j}}$.

Table 3 summarizes the values of the intervals $\hat{a}_{j}, \hat{b}_{j}$ for cost function and $\hat{c}_{j}, \hat{d}_{j}$ for time function, which are found through interpolation as discussed above. In Step 0, it is not possible to remove the pollutants. So $\hat{a}_{j}, \hat{b}_{j}, \hat{c}_{j}, \hat{d}_{j}$ are $\hat{0}$, where $\hat{0}=[0,0]$. The total cost and total time taken for completion of

Table 3. Evaluated $\hat{a}_{j}, \hat{b}_{j}, \hat{c}_{j}, \hat{d}_{j}$.

\begin{tabular}{ccccc}
\hline$j$ & $\hat{a}_{j}$ & $\hat{b}_{j}$ & $\hat{c}_{j}$ & $\hat{d}_{j}$ \\
\hline 1 & {$[-0.672,-0.450]$} & {$[0.386,0.726]$} & {$[-0.661,-0.483]$} & {$[0.490,0.767]$} \\
2 & {$[-0.786,-0.660]$} & {$[0.407,0.585]$} & {$[-1.118,-0.930]$} & {$[0.224,0.356]$} \\
3 & {$[-0.482,-0.416]$} & {$[0.875,1.343]$} & {$[-0.488,-0.394]$} & {$[1.259,1.592]$} \\
\hline
\end{tabular}

all the steps for this system are respectively

$$
\begin{aligned}
\hat{Y}(x) \triangleq[0.386,0.726] \odot x_{1}^{[-0.672,-0.450]} & \oplus[0.407,0.585] \odot x_{2}^{[-0.786,-0.660]} \\
& \oplus[0.875,1.343] \odot x_{3}^{[-0.482,-0.416]}
\end{aligned}
$$

and

$$
\begin{aligned}
\hat{T}(x) \triangleq[0.490,0.767] \odot x_{1}^{[-0.661,-0.483]} & \oplus[0.224,0.356] \odot x_{2}^{[-1.118,-0.930]} \\
& \oplus[1.259,1.592] \odot x_{3}^{[-0.488,-0.394]} .
\end{aligned}
$$

$\hat{Y}(x)$ and $\hat{T}(x)$ are the interval valued functions which have to be minimized simultaneously. Generally, after waste water is purified through $j$ steps, the remaining amount of the water pollutant (percentage) in water is acceptable up 
to some desirable limit say $b(b>0)$. In Step II $x_{1} \%$ of pollutant is entering and it is possible to remove $x_{2} \%$ of pollutants. So, after completion of this step the remaining amount of pollutant is $x_{1} x_{2} \%$. Similarly after Step III, it is $x_{1} x_{2} x_{3} \%$. We assume that after completion of these steps the remaining amount of water pollutant is limited up to $b \%(b>0)$. So, $x_{1} x_{2} x_{3} \leq b$. For $p=96$, that is, if at least $96 \%$ of the pollutants has to be removed then, $b=1-0.96=0.04$.

Summarizing the above discussion, the waste water treatment optimization model denoted by $(W-I M G P)$ can be formulated as

$$
\begin{aligned}
& (W-I M G P): \\
& \quad \min \left\{[0.386,0.726] \odot x_{1}^{[-0.672,-0.450]} \oplus[0.407,0.585] \odot x_{2}^{[-0.786,-0.660]}\right. \\
& \quad \oplus[0.875,1.343] \odot x_{3}^{[-0.482,-0.416]}, \\
& {[0.490,0.767] \odot x_{1}^{[-0.661,-0.483]} \oplus[0.224,0.356] \odot x_{2}^{[-1.118,-0.930]}} \\
& \left.\quad \oplus[1.259,1.592] \odot x_{3}^{[-0.488,-0.394]}\right\} \\
& \text { subject to } x_{1} x_{2} x_{3} \leq 0.04,0<x_{j} \leq 1 .
\end{aligned}
$$

This is an interval multi-objective geometric programming (IMGP) model.

\subsection{Solution of $(W-I M G P)$}

In $(W-I M G P)$ model,

$$
\begin{aligned}
& g_{1}(x)=x_{1} x_{2} x_{3}, S=\left\{\left(x_{1}, x_{2}, x_{3}\right): x_{1} x_{2} x_{3} \leq 0.04,0<x_{j} \leq 1\right\}, \\
& f_{1}^{L}(x)=0.386 x_{1}^{-0.450}+0.407 x_{2}^{-0.660}+0.875 x_{3}^{-0.416}, \\
& f_{1}^{R}(x)=0.726 x_{1}^{-0.672}+0.585 x_{2}^{-0.786}+1.343 x_{3}^{-0.482}, \\
& f_{2}^{L}(x)=0.490 x_{1}^{-0.483}+0.224 x_{2}^{-0.930}+1.259 x_{3}^{-0.394}, \\
& f_{2}^{R}(x)=0.767 x_{1}^{-0.661}+0.356 x_{2}^{-1.118}+1.592 x_{3}^{-0.488} .
\end{aligned}
$$

Based on the above information, $\chi$-efficient solution of this model can be found using the proposed methodology in Section 3. Details may be avoided. Here $S^{\prime}=S$. Suppose goals for the functions $\left[f_{1}^{L}(x), f_{1}^{R}(x)\right]$ and $\left[f_{2}^{L}(x), f_{2}^{R}(x)\right]$ are $[4,7]$ and $[6,8]$ respectively (provided by decision maker). So $\chi_{i}^{O}$, for $i=\{1,2\}$ are as follows

$$
\begin{aligned}
& \chi_{1}^{O}\left(\hat{f}_{1}(x),[4,7]\right)=\frac{7-f_{1}^{L}(x)}{3+\left(f_{1}^{R}(x)-f_{1}^{L}(x)\right)}, \\
& \chi_{2}^{O}\left(\hat{f}_{2}(x),[6,8]\right)=\frac{8-f_{2}^{L}(x)}{2+\left(f_{2}^{R}(x)-f_{2}^{L}(x)\right)} .
\end{aligned}
$$

The inequality $\theta \leq \chi_{1}^{O}\left(\hat{f}_{1}(x),[4,7]\right)$ is equivalent to

$$
0.386 x_{1}^{-0.450}+0.407 x_{2}^{-0.660}+0.875 x_{3}^{-0.416}+\left(3+f_{1}^{R}(x)-f_{1}^{L}(x)\right) \theta \leq 7,
$$

and the inequality $\theta \leq \chi_{2}^{O}\left(\hat{f}_{2}(x),[6,8]\right)$ is equivalent to

$$
0.490 x_{1}^{-0.483}+0.224 x_{2}^{-0.930}+1.259 x_{3}^{-0.394}+\left(2+f_{2}^{R}(x)-f_{2}^{L}(x)\right) \theta \leq 8 .
$$


Here $(I M G P)^{\prime}$ becomes the deterministic equivalent $(W-I M G P)^{\prime}$, which is

$$
\begin{aligned}
& (W-I M G P)^{\prime}: \max \theta \\
& \quad \text { subject to }(1-\theta)\left(0.055 x_{1}^{-0.450}+0.058 x_{2}^{-0.660}+0.125 x_{3}^{-0.416}\right) \\
& \quad+\theta\left(0.104 x_{1}^{-0.672}+0.083 x_{2}^{-0.786}+0.192 x_{3}^{-0.482}\right)+0.429 \theta \leq 1, \\
& (1-\theta)\left(0.061 x_{1}^{-0.483}+0.028 x_{2}^{-0.930}+0.157 x_{3}^{-0.394}\right) \\
& \quad+\theta\left(0.096 x_{1}^{-0.661}+0.045 x_{2}^{-1.118}+0.199 x_{3}^{-0.488}\right)+0.250 \theta \leq 1, \\
& 25 x_{1} x_{2} x_{3} \leq 1,0<x_{1}, x_{2}, x_{3}<1, \frac{1}{2}<\theta<1 .
\end{aligned}
$$

The transformed model is a geometric programming problem with positive degree of difficulty. Optimal solution of the problem is found using GGPLAB ( [23]) optimization environment in MATLAB R2012a as

$$
x_{1}^{o p t}=0.300, x_{2}^{o p t}=0.352, x_{3}^{o p t}=0.379, \theta^{o p t}=0.793 .
$$

Since the feasible region of the problem is a deterministic region. So the degree of acceptability/feasibility of the solution is 1 . Both the objectives satisfy their goals with degree of flexibility 0.793 . Hence a $\chi$-efficient solution of the given interval multi-objective problem is $x^{\text {opt }}=\left(\begin{array}{lll}0.300 & 0.352 & 0.379\end{array}\right)$ with $79.3 \%$ degree of satisfaction. In this case $96 \%$ of the pollutant is removable. $\left(x_{1}^{o p t}, x_{2}^{o p t}, x_{3}^{o p t}\right.$ are remaining amount of pollutant in corresponding steps.)

Note 2. In this model the parameter $b$ represents the desirable limit of remaining water pollutants after completion of all the process of waste water treatment system. Other parameters of the model are uncertain. For any change on the pollutant limit $b$ to $b \pm \epsilon$, the constraint for remaining amount of pollutant in $(W-I M G P)^{\prime}$ becomes $x_{1} x_{2} x_{3} \leq(b \pm \epsilon) \%$. In that case the sensitivity analysis of the model $(W-I M G P)$ can be studied using the sensitivity analysis technique of general geometric programming problem, since $(W-I M G P)^{\prime}$ is a general geometric programming problem. Corresponding to different values of $b$, change in the solution as well as change in the range of total cost and total time taken for the completion of all the steps for the system are provided in Table 4 .

Table 4. Optimal solutions corresponding to different desirable limits of remaining pollutants $b$.

\begin{tabular}{cccccc}
\hline$b$ & $x_{1}$ & $x_{2}$ & $x_{3}$ & Total cost $\hat{Y}(x)$ & Total time taken $\hat{T}(x)$ \\
\hline 0.04 & 0.300 & 0.352 & 0.379 & {$[2.783,5.103]$} & {$[3.313,5.400]$} \\
0.05 & 0.324 & 0.374 & 0.412 & {$[2.684,4.873]$} & {$[3.188,5.137]$} \\
0.03 & 0.315 & 0.348 & 0.364 & {$[2.916,5.418]$} & {$[3.482,5.759]$} \\
0.02 & 0.230 & 0.300 & 0.289 & {$[3.113,5.896]$} & {$[3.734,6.307]$} \\
0.01 & 0.175 & 0.255 & 0.223 & {$[3.479,6.816]$} & {$[4.206,7.371]$} \\
\hline
\end{tabular}

\section{Conclusions}

In this paper a new partial ordering is introduced to compare interval vectors. Existence of solution of a general interval geometric programming problem is 
studied and summarized in a theorem. A methodology is derived for the solution of the model. In the process of the methodology, one may observe that $\chi$-efficient solution of $(I M G P)$ is associated with certain degree of feasibility and certain degree of flexibility of the objective functions towards the goals. A possible application of this methodology is discussed in a waste water treatment system. The methodology provides compromise lower and upper bound of minimum cost as well as minimum time requirement of the waste water treatment model. The model is explained in hypothetical data. However real life waste water model can also be studied for large data, which is beyond the scope of this theoretical development. Study of sensitivity analysis on the lower and upper bounds of the interval parameters of a general interval geometric programming problem $(I M G P)$ is complex. We leave this for future study.

\section{Acknowledgement}

The authors are greatly indebted to the anonymous referees for valuable comments and remarks.

\section{Appendix}

\section{Proof of Lemma 1:}

For this we need to show $\preceq_{\chi}$ is $(i)$ reflexive, $(i i)$ antisymmetric and (iii) transitive.

Reflexive : Suppose $\hat{a} \in I(\mathbb{R})$. Since $\mu(\hat{a})=\mu(\hat{a})$ and $\chi(\hat{a}, \hat{a})=\frac{1}{2}$, so $\hat{a} \preceq_{\chi} \hat{a}$. Hence $\preceq_{\chi}$ is a reflexive relation.

Antisymmetric : Consider $\hat{a}, \hat{b} \in I(\mathbb{R})$ and $\hat{a} \preceq_{\chi} \hat{b}$ and $\hat{b} \preceq_{\chi} \hat{a}$. $\hat{a} \preceq_{\chi} \hat{b}$ implies $\mu(\hat{a}) \leq \mu(\hat{b})$ and $\chi(\hat{a}, \hat{b}) \in\left[\frac{1}{2}, 1\right]$. Again $\hat{b} \preceq_{\chi} \hat{a}$ implies $\mu(\hat{b}) \leq \mu(\hat{a})$ and $\chi(\hat{b}, \hat{a}) \in\left[\frac{1}{2}, 1\right]$. Hence $\mu(\hat{a})=\mu(\hat{b}) . \chi(\hat{a}, \hat{b}) \in\left[\frac{1}{2}, 1\right]$ implies $\chi(\hat{a}, \hat{b}) \geq \chi(\hat{b}, \hat{a})$, and $\chi(\hat{a}, \hat{b}) \in\left[\frac{1}{2}, 1\right]$ implies $\chi(\hat{b}, \hat{a}) \geq \chi(\hat{a}, \hat{b})$. Hence $\chi(\hat{a}, \hat{b})=\chi(\hat{b}, \hat{a})$. Because $\chi(\hat{a}, \hat{b})+\chi(\hat{b}, \hat{a})=1$ and $\chi(\hat{a}, \hat{b})=\chi(\hat{b}, \hat{a})$, so $\chi(\hat{a}, \hat{b})=\chi(\hat{b}, \hat{a})=\frac{1}{2}$. Since $\mu(\hat{a})=\mu(\hat{b})$ and $\chi(\hat{a}, \hat{b})=\chi(\hat{b}, \hat{a})=\frac{1}{2}$, so $\hat{a}=\hat{b}$. Hence $\preceq_{\chi}$ is an antisymmetric relation.

Transitive : For $\hat{a}, \hat{b}, \hat{c} \in I(\mathbb{R})$, consider $\hat{a} \preceq_{\chi} \hat{b}$ and $\hat{b} \preceq_{\chi} \hat{c}$. $\hat{a} \preceq_{\chi} \hat{b}$ implies $\mu(\hat{a}) \leq \mu(\hat{b})$ and $\chi(\hat{a}, \hat{b}) \in\left[\frac{1}{2}, 1\right] . \quad \hat{b} \preceq_{\chi} \hat{c}$ implies $\mu(\hat{b}) \leq \mu(\hat{c})$ and $\chi(\hat{b}, \hat{c}) \in\left[\frac{1}{2}, 1\right]$. Hence $\mu(\hat{a}) \leq \mu(\hat{c}) . \chi(\hat{a}, \hat{b}) \in\left[\frac{1}{2}, 1\right]$ implies $\chi(\hat{a}, \hat{b}) \geq \chi(\hat{b}, \hat{a})$. Hence $b^{R}+b^{L} \geq a^{R}+a^{L}$. Also, $\chi(\hat{b}, \hat{c}) \in\left[\frac{1}{2}, 1\right]$ implies $\chi(\hat{b}, \hat{c}) \geq \chi(\hat{c}, \hat{b})$. Hence $c^{R}+c^{L} \geq b^{R}+b^{L}$. From both the relations we have $c^{R}+c^{L} \geq a^{R}+a^{L}$. This implies, $c^{R}-a^{L} \geq a^{R}-c^{L}$. So $\frac{c^{R}-a^{L}}{\mu(\hat{c})+\mu(\hat{a})} \geq \frac{a^{R}-c^{L}}{\mu(\hat{c})+\mu(\hat{a})}$, which implies $\chi(\hat{a}, \hat{c}) \geq \chi(\hat{c}, \hat{a})$. This means, $\chi(\hat{a}, \hat{c}) \in\left[\frac{1}{2}, 1\right]$. So $\hat{a} \preceq_{\chi} \hat{c}$. Hence $\preceq_{\chi}$ is a transitive relation.

This implies that $\preceq_{\chi}$ is a partial order relation.

Proof of Theorem 1: Here $\theta^{\text {opt }}=\theta\left(x^{o p t}\right)$.

Denote $\quad \hat{f}_{i}(x)=\widehat{\sum}_{t_{i}=1}^{s_{0 i}} \hat{c}_{0 t_{i}} \widehat{\prod}_{l=1}^{n} x_{l}^{\hat{\gamma}_{0 l i}}, \quad f_{i}^{L}(x)=\sum_{t_{i}=1}^{s_{0 i}} c_{0 t_{i}}^{L} \prod_{l=1}^{n} x_{l}^{\eta_{0 l i}}$, 
$f_{i}^{R}(x)=\sum_{t_{i}=1}^{s_{0 i}} c_{0 t_{i}}^{R} \prod_{l=1}^{n} x_{l}^{\eta_{0 l i}}, \hat{g}_{j}(x)={\widehat{\sum_{t=1}^{s}}}_{s_{j}} \hat{c}_{j t} \widehat{\prod}_{l=1}^{n} x_{l}^{\hat{\gamma}_{j t l}}$. Suppose $x^{o p t}$ is not an $\chi$-efficient solution. So from Definition 4 , we can say that there exists $x^{*} \neq x^{o p t}$, which is a feasible solution of $(I M G P)$ with degree of acceptability $\tau^{*}$ such that $\hat{f}_{i}\left(x^{*}\right) \prec_{\chi} \hat{f}_{i}\left(x_{o p t}\right), \forall i \in \Lambda_{k}$. Hence $\mu\left(\hat{f}_{i}\left(x^{*}\right)\right) \leq \mu\left(\hat{f}_{i}\left(x^{o p t}\right)\right)$ and $f_{i}^{R}\left(x^{*}\right) \leq f_{i}^{L}\left(x^{o p t}\right)$. This implies $f_{i}^{L}\left(x^{*}\right) \leq f_{i}^{R}\left(x^{*}\right) \leq f_{i}^{L}\left(x^{o p t}\right) \leq f_{i}^{R}\left(x^{o p t}\right)$. So

$$
u_{i}-f_{i}^{L}\left(x^{*}\right) \geq u_{i}-f_{i}^{L}\left(x^{o p t}\right) \geq 0 .
$$

Since $\left[l_{i}, u_{i}\right]$ are non degenerate intervals, we have

$$
0<\left(u_{i}-l_{i}\right)+\left(f_{i}^{R}\left(x^{*}\right)-f_{i}^{L}\left(x^{*}\right)\right) \leq\left(u_{i}-l_{i}\right)+\left(f_{i}^{R}\left(x^{o p t}\right)-f_{i}^{L}\left(x^{o p t}\right)\right) .
$$

From (5.1) and (5.2), we get

$$
\frac{u_{i}-f_{i}^{L}\left(x^{*}\right)}{\left(u_{i}-l_{i}\right)+\left(f_{i}^{R}\left(x^{*}\right)-f_{i}^{L}\left(x^{*}\right)\right)} \geq \frac{u_{i}-f_{i}^{L}\left(x^{o p t}\right)}{\left(u_{i}-l_{i}\right)+\left(f_{i}^{R}\left(x^{o p t}\right)-f_{i}^{L}\left(x^{o p t}\right)\right)} .
$$

Also we have

$$
\theta^{o p t} \leq \frac{u_{i}-f_{i}^{L}\left(x^{o p t}\right)}{\left(u_{i}-l_{i}\right)+\left(f_{i}^{R}\left(x^{o p t}\right)-f_{i}^{L}\left(x^{o p t}\right)\right)} \leq \frac{u_{i}-f_{i}^{L}\left(x^{*}\right)}{\left(u_{i}-l_{i}\right)+\left(f_{i}^{R}\left(x^{*}\right)-f_{i}^{L}\left(x^{*}\right)\right)} .
$$

$x^{*} \in S$ implies that $x^{*}$ is a feasible point with some degree of acceptability $\tau^{*} \in\left[\frac{1}{2}, 1\right]$, where $\tau^{*}=\min _{j}\left\{\frac{b_{j}^{R}-g_{j}^{L}(x)}{\left(b_{j}^{R}-b_{j}^{L}\right)+\left(g_{j}^{R}(x)-g_{j}^{L}(x)\right)}\right\}$. So $\left(x^{*}, \tau^{*}\right)$ is a feasible point of $(I M G P)^{\prime}$. Now,

$$
\min _{i \in \Lambda_{k}}\left\{\frac{u_{i}-f_{i}^{L}\left(x^{*}\right)}{\left(u_{i}-l_{i}\right)+\left(f_{i}^{R}\left(x^{*}\right)-f_{i}^{L}\left(x^{*}\right)\right)}\right\} \geq \min _{i \in \Lambda_{k}}\left\{\frac{u_{i}-f_{i}^{L}\left(x^{o p t}\right)}{\left(u_{i}-l_{i}\right)+\left(f_{i}^{R}\left(x^{o p t}\right)-f_{i}^{L}\left(x^{o p t}\right)\right)}\right\} .
$$

Hence $\theta\left(x^{*}\right) \geq \theta\left(x^{o p t}\right)$. If $\theta\left(x^{*}\right)=\theta\left(x^{o p t}\right)$ then $x^{*}$ and $x^{o p t}$ are alternate optimal solutions of $(I M G P)^{\prime}$ with degree of feasibility $\tau^{*}$ and $x^{*}$ is a $\chi^{-}$ efficient solution of $(I M G P) . \theta\left(x^{*}\right)>\theta\left(x^{o p t}\right)$ leads to a contradiction to the optimality of $x^{\text {opt }}$.

\section{References}

[1] A.K. Bhurjee and G. Panda. Efficient solution of interval optimization problem. Mathematical Methods of Operations Research, 76(3):273-288, 2012. http://dx.doi.org/10.1007/s00186-012-0399-0.

[2] S. Chanas and D. Kuchta. Multiobjective programming in optimization of interval objective functions A generalized approach. European Journal of Operational Research, 94(3):594-598, 1996. http://dx.doi.org/10.1016/0377-2217(95)000550 .

[3] J.J. Dinkel and M.J. Tretter. An interval arithmetic approach to sensitivity analysis in geometric programming. Operations Research, 35(6):859-866, 1987. http://dx.doi.org/10.1287/opre.35.6.859.

[4] J.G. Ecker. A geometric programming model for optimal allocation of stream dissolved oxygen. Management Science, 21(6):658-668, 1975. http://dx.doi.org/10.1287/mnsc.21.6.658. 
[5] L. Hammadi, A. Ponton and M. Belhadri. Rheological study and valorization of waste sludge from wastewater treatment plants in the dredging operation of hydraulic dams. Energy Procedia, 6:302-309, 2011. http://dx.doi.org/10.1016/j.egypro.2011.05.034.

[6] E. Hansen and G.W. Walster. Global optimization using interval analysis. Marcel Dekker Inc, New York, 2004.

[7] M. Hladík. Optimal value range in interval linear programming. Fuzzy Optimization and Decision Making, 8(3):283-294, 2009. http://dx.doi.org/10.1007/s10700-009-9060-7.

[8] M. Hladík. Optimal value bounds in nonlinear programming with interval data. TOP, 19(1):93-106, 2011. http://dx.doi.org/10.1007/s11750-009-0099-y.

[9] B.Q. $\mathrm{Hu}$ and S. Wang. A novel approach in uncertain programming part I: New arithmetic and order relation for interval numbers. Journal of Industrial and Management Optimization, 2(4):351-371, 2006. http://dx.doi.org/10.3934/jimo.2006.2.351.

[10] H. Ishibuchi and H. Tanaka. Multiobjective programming in optimization of the interval objective function. European Journal of Operational Research, 48(2):219-225, 1990. http://dx.doi.org/10.1016/0377-2217(90)90375-L.

[11] M. Jana and G. Panda. Solution of nonlinear interval vector optimization problem. Operational Research, 14(1):71-85, 2014. http://dx.doi.org/10.1007/s12351-013-0137-2.

[12] A. Jayswal, I. Stancu-Minasian and I. Ahmad. On sufficiency and duality for a class of interval-valued programming problems. Applied Mathematics and Computation, 218(8):4119-4127, 2011. http://dx.doi.org/10.1016/j.amc.2011.09.041.

[13] C. Jiang, X. Han, G.R. Liu and G.P. Liu. A nonlinear interval number programming method for uncertain optimization problems. European Journal of Operational Research, 188(1):1-13, 2008. http://dx.doi.org/10.1016/j.ejor.2007.03.031.

[14] J.C. Liebman and W.R. Lynn. The optimal allocation of stream dissolved oxygen. Water Resources Research, 2(3):581-591, 1966. http://dx.doi.org/10.1029/WR002i003p00581.

[15] S.T. Liu. Posynomial geometric programming with parametric uncertainty. European journal of Operational Research, 168(2):345-353, 2006. http://dx.doi.org/10.1016/j.ejor.2004.04.046.

[16] S.T. Liu. Posynomial geometric programming with interval exponents and coefficients. European Journal of Operational Research, 186(1):17-27, 2008. http://dx.doi.org/10.1016/j.ejor.2007.01.031.

[17] S.T. Liu. Using geometric programming to profit maximization with interval coefficients and quantity discount. Applied Mathematics and Computation, 209(2):259-265, 2009. http://dx.doi.org/10.1016/j.amc.2008.12.035.

[18] S.T. Liu and R.T. Wang. A numerical solution method to interval quadratic programming. Applied Mathematics and Computation, 189(2):1274-1281, 2007. http://dx.doi.org/10.1016/j.amc.2006.12.007.

[19] D.P. Loucks, C.S. Revelle and W.R. Lynn. Linear progamming models for water pollution control. Management Science, 14(4):B-166-B-181, 1967. http://dx.doi.org/10.1287/mnsc.14.4.B166. 
[20] G.S. Mahapatra and T.K. Mandal. Posynomial parametric geometric programming with interval valued coefficient. Journal of Optimization Theory and Applications, 154:120-132, 2012. http://dx.doi.org/10.1007/s10957-012-9996-6.

[21] I. Merino, L.F. Arévalo and F. Romero. Characterization and possible uses of ashes from wastewater treatment plants. Waste management, 25(10):1046-1054, 2005. http://dx.doi.org/10.1016/j.wasman.2004.12.023.

[22] R.E. Moore, R.B. Kearfott and M.J. Cloud. Introduction to interval analysis. Society for Industrial and Applied Mathematics, 2009. ISBN 9780898717716.

[23] A. Mutapcic, K. Koh, S. Kim and S. Boyd. Ggplab version 1.00 a matlab toolbox for geometric programming, 2006.

[24] M. Novak and P. Horvat. Mathematical modelling and optimisation of a waste water treatment plant by combined oxygen electrode and biological waste water treatment model. Applied Mathematical Modelling, 36(8):3813-3825, 2012. http://dx.doi.org/10.1016/j.apm.2011.11.028.

[25] X. Qin, G. Huang, B. Chen and B. Zhang. An interval-parameter waste-loadallocation model for river water quality management under uncertainty. Environmental management, 43(6):999-1012, 2009. http://dx.doi.org/10.1007/s00267009-9278-8.

[26] A. Sengupta and T.K. Pal. On comparing interval numbers. European Journal of Operational Research, 127(1):28-43, 2000. http://dx.doi.org/10.1016/S03772217(99)00319-7.

[27] Y. Smeers and D. Tyteca. A geometric programming model for the optimal design of wastewater treatment plants. Operations Research, 32(2):314-342, 1984. http://dx.doi.org/10.1287/opre.32.2.314.

[28] H.C. Wu. On interval-valued nonlinear programming problems. Journal of Mathematical Analysis and Applications, 338(1):299-316, 2008. http://dx.doi.org/10.1016/j.jmaa.2007.05.023.

[29] S. Yordanova and N. Noikova. An investigation of the model of aerobic waste water treatment processes. Bioprocess Engineering, 15(4):201-203, 1996. http://dx.doi.org/10.1007/BF00369482. 